\title{
Distinct polymer physics principles govern chromatin dynamics in mouse and Drosophila topological domains
}

\author{
Vuthy Ea ${ }^{1}$, Tom Sexton ${ }^{2}$, Thierry Gostan ${ }^{1}$, Laurie Herviou' ${ }^{1}$, Marie-Odile Baudement ${ }^{1}$, Yunzhe Zhang ${ }^{3}$, Soizik Berlivet ${ }^{1}$, \\ Marie-Noëlle Le Lay-Taha', Guy Cathala', ${ }^{1,4}$ Annick Lesne ${ }^{1,4,5}$, Jean-Marc Victor ${ }^{1,4,5}$, Yuhong Fan ${ }^{3}$, Giacomo Cavalli, ${ }^{2,4}$ \\ and Thierry Forné ${ }^{14^{*}}$
}

\begin{abstract}
Background: In higher eukaryotes, the genome is partitioned into large "Topologically Associating Domains" (TADs) in which the chromatin displays favoured long-range contacts. While a crumpled/fractal globule organization has received experimental supports at higher-order levels, the organization principles that govern chromatin dynamics within these TADs remain unclear. Using simple polymer models, we previously showed that, in mouse liver cells, gene-rich domains tend to adopt a statistical helix shape when no significant locus-specific interaction takes place.

Results: Here, we use data from diverse 3C-derived methods to explore chromatin dynamics within mouse and Drosophila TADs. In mouse Embryonic Stem Cells (mESC), that possess large TADs (median size of $840 \mathrm{~kb}$ ), we show that the statistical helix model, but not globule models, is relevant not only in gene-rich TADs, but also in gene-poor and gene-desert TADs. Interestingly, this statistical helix organization is considerably relaxed in mESC compared to liver cells, indicating that the impact of the constraints responsible for this organization is weaker in pluripotent cells. Finally, depletion of histone $\mathrm{H} 1$ in mESC alters local chromatin flexibility but not the statistical helix organization. In Drosophila, which possesses TADs of smaller sizes (median size of $70 \mathrm{~kb}$ ), we show that, while chromatin compaction and flexibility are finely tuned according to the epigenetic landscape, chromatin dynamics within TADs is generally compatible with an unconstrained polymer configuration.
\end{abstract}

Conclusions: Models issued from polymer physics can accurately describe the organization principles governing chromatin dynamics in both mouse and Drosophila TADs. However, constraints applied on this dynamics within mammalian TADs have a peculiar impact resulting in a statistical helix organization.

Keywords: Chromatin dynamics, Polymer models, Topological domains, Epigenetics, H1 histone

\section{Background}

During the last decade, the advent of Chromosome Conformation Capture (3C) [1] and its derived technologies $(4 \mathrm{C}, 5 \mathrm{C}, \mathrm{Hi}-\mathrm{C})$ [2] allowed to explore genome organization with unprecedented resolution and accuracy. By capturing all chromatin contacts present at a given time in their physiological nuclear context, and then by averaging these events over several millions of cells, the quantitative $3 \mathrm{C}$

\footnotetext{
* Correspondence: forne@igmm.cnrs.fr

'Institut de Génétique Moléculaire de Montpellier, UMR5535, CNRS,

Université de Montpellier, 1919 Route de Mende, 34293 Montpellier, Cedex

5, France

${ }^{4}$ CNRS GDR 3536 UPMC, Sorbonne universités, Paris, France

Full list of author information is available at the end of the article
}

method [3] allows to access the relative contact frequencies between chromatin segments in vivo. This feature is key to understanding chromatin dynamics in vivo because it depends not only on fundamental biophysical parameters of the chromatin (such as compaction and stiffness) that determine its local organization at the nucleosomal scale, but also on constraints that impact its higher-order/supranucleosomal organization. These latter constraints can result either from nuclear determinants that organize chromatin at higher scales ("top-down" constraints) or from some intrinsic locus-specific components of the chromatin that are controlling genomic functions, like epigenetic 
modifications or the binding of specific factors ("bottomup" constraints) [4].

$\mathrm{Hi}-\mathrm{C}$ approaches (that combine $3 \mathrm{C}$ assays with highthroughput sequencing) provided genome-wide profiling of contact frequencies in the yeast (Saccharomyces cerevisiae) [5], fly (Drosophila melanogaster) [6], mouse (Mus musculus domesticus) [7] and human [8,9] genomes. While these data confirmed that higher-order chromatin dynamics appears to be globally unconstrained in yeast, they showed that this organization level is constrained in higher eukaryotes where the chromatin is compartmentalized into chromosomal territories that are themselves further partitioned into the so-called "Topologically Associating Domains" (TADs) [10] or contact domains [9]. TADs and contact domains are defined as chromosomal sub-compartments that display preferential contacts in cis. However, they are restricted to interphase cells and disappear in mitotic chromosomes [11], to be re-acquired in the early G1 phase [12]. They are physically delimited by borders that are gene-rich regions enriched in specific factors like the insulator protein CTCF $[7,9,13,14]$. Noticeably, the location of TAD borders appears to be quite stable across cell types. It is commonly accepted that, within TADs, chromatin is organized into chromatin loops, via locus-specific interactions, and that this organization is tightly related to genome function [9, 15-17]. It has recently been evidenced that such interactions occur in the context of fluctuating structures rather than being stable loops [18], and we previously showed that, in the absence of strong long-range locus-specific interactions, this underlying dynamics of the chromatin undergo constraints in gene-rich regions resulting in modulated contact frequencies over large genomic distances [4]. While the involvement of locus-specific factors in chromatin-loop formation, within TADs, is now well established [9], the physical properties that govern the underlying chromatin dynamics at that scale remains unknown.

Here, using quantitative $3 \mathrm{C}$ experiments, we report that the modulation of contact frequencies previously described in liver cells [4] is also present in pluripotent mouse Embryonic Stem Cells (mESC), not only in generich TADs, but also in gene-poor and gene-desert domains. Therefore, the constraints that affect higher-order chromatin dynamics in mammals appear to widely affect TADs in diverse genomic contexts. We show that the equilibrium/crumpled globule models do not reproduce chromatin dynamics within mammalian TADs. In contrast, models derived from polymer physics can accurately describe chromatin dynamics at that scale in both mouse and Drosophila TADs. In the mouse, we found that chromatin dynamics is less constrained in ESC than in liver cells, and that this constraint is also strongly attenuated in a TAD spanning a gene-desert compared to gene-poor or gene-rich TADs. In Drosophila melanogaster, using Hi-C data obtained from embryos, we show that, on a local scale, chromatin dynamics is finely tuned according to the epigenetic landscape: the nucleofilament is less compact and more flexible in active than in heterochromatic domains. However, in contrast to mammals, the higherorder chromatin dynamics in Drosophila appears largely unconstrained.

\section{Results}

To explore the influence of the genomic context on chromatin dynamics, we first investigated mouse ESC, for which TADs have been finely defined [7]. We focused on three types of domains: five gene-rich TADs, two genepoor TADs [19] and one gene-desert TAD (Additional file 1a and $\mathrm{b}$ ). The regions investigated in the two gene-poor TADs (Additional file 1b) are devoid of any known genes or putative regulatory elements, and their homozygous deletion in mouse results in fully viable pups, with no obvious alteration [19]. These TADs actually do contain several genes, but the closest from the regions analysed are located around $300 \mathrm{~kb}$ away. In contrast, the genedesert TAD is containing a single gene located more than $1.5 \mathrm{Mb}$ away from the region analysed (Additional file 1a).

\section{Equilibrium/crumpled globule models do not reproduce chromatin dynamics within mammalian TADs}

Equilibrium and crumpled/fractal globule models, have been developed to describe chromatin dynamics in vivo. It was shown that, when one looks at decreasing contact frequencies as a function of increasing genomic distances in a Log-Log plot, the equilibrium globule model follows a power-law scaling associated to a slope of $-3 / 2$ over two orders of magnitude while the crumpled globule model has a slope of -1 [20]. Using $\mathrm{Hi}-\mathrm{C}$ data, it was shown that crumpled globule features are characteristic of chromatin dynamics above $1 \mathrm{Mb}$ (chromosome territory/inter-TADs dynamics) but that they may not be valid for separation distances shorter than $100 \mathrm{~kb}$ [8].

To assess whether such organization principles apply to chromatin dynamics within TADs, we thus performed quantitative $3 \mathrm{C}$ experiments in the different TADs described above and, using Log-Log plots, we showed that gene-rich, as well as gene-poor and gene-desert TADs display slopes superior to $-1(-0.60$ to -0.48$)$ (Fig. 1) which are incompatible with the equilibrium or crumpled globule models. Therefore, neither the equilibrium nor the crumpled globule models accurately reproduce chromatin dynamics within mammalian TADs.

\section{Chromatin dynamics is less constrained in pluripotent mESC than in liver cells}

We then fitted our data to two models, derived from polymer physics, that were previously used to describe 


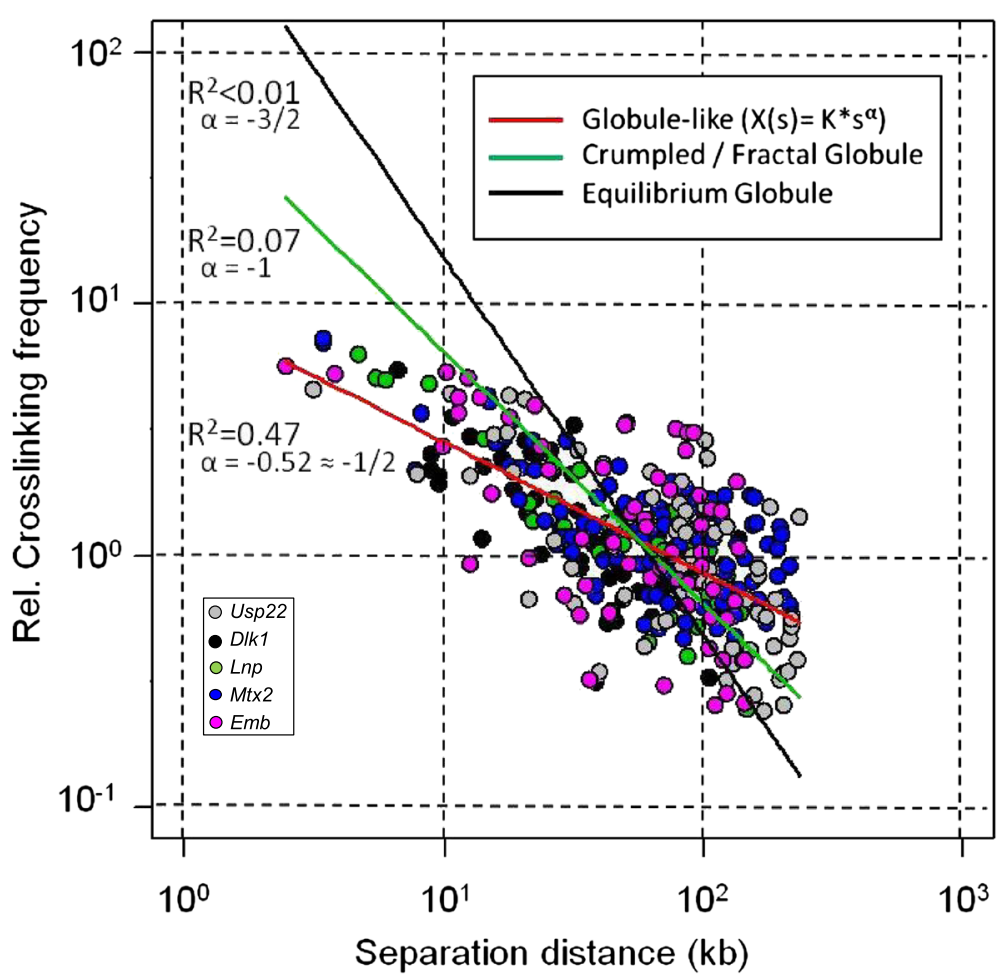

Fig. 1 Fitting globule models to contact frequencies quantified in mESC. Experimental 3C-qPCR data obtained for wt mESC in gene-rich TADs (Fig. 2) have been displayed into a Log-Log plot and globule models were fitted to the following power-law: $X(s)=k^{*} s^{a}$ (adapted from Eq. 6 and Eq. 9 from ref. [20]), where $X(s)$ is the cross-linking frequency, $s$ (in kb) is the site separation along the genome, $K$ is representing the efficiency of cross-linking and the exponent $a$ is the slope associated to this power-law. Best-fits (using the nls object of the $R$ software) show that the slope associated to our experimental data (red line) is approximately $a=-1 / 2(-0.52)$ with a correlation coefficient $R^{2}=0.47$, while correlation coefficients associated to the equilibrium $(a=-3 / 2)$ (black line) or crumpled globules ( $a=-1)$ (green line) are much lower

chromatin dynamics in the yeast Saccharomyces cerevisiae [1, 21] and in mammals [4]. The first model [see equations (eqs.) 1 and 2 in Methods] provides measurements of three key parameters of local chromatin dynamics (nucleosomal scale): $K$ reflects features of the experimental setting (mainly cross-linking efficiency); $L$ is the length of a chromatin segment (in $\mathrm{nm}$ ) containing $1 \mathrm{~kb}$ of genomic DNA, thus reflecting chromatin compaction (in $\mathrm{nm} / \mathrm{kb}$ ); $S$ (the Kuhn's statistical segment, in $\mathrm{kb}$ ) is a measure of chromatin flexibility $[1,21]$. The higher cross-linking efficiency, chromatin compaction and flexibility, the lower the values of parameters $K, L$ and $S$ will be. This model assumes that, at higher-order organization levels, chromatin does not undergo any special constraints. Therefore, we define it as "unconstrained chromatin" model. The second model is named "statistical helix" model. It provides measurements of the same parameters of local chromatin dynamics, but it also takes into account constraints that may impact chromatin dynamics at the higher-order level (supranucleosomal scale). In this model, the higher-order chromatin dynamics is described as if constraints imposed onto chromatin were folding, statistically, the chromatin into a helical shape that can be characterized by two parameters: its mean Diameter $(D)$ (in $\mathrm{nm}$ ) and its mean Pitch $(P)$ (in $\mathrm{nm}$ ) [eq.2] [4]. These two parameters are thus describing the presence of constraints that impact higher-order chromatin dynamics. The weaker the effects of the constraints, the less pronounced the parameters of the statistical helix will be (i.e. large diameter and/or large Pitch).

As previously found in mouse liver cells [4], gene-rich TADs display modulated contact frequencies and the statistical helix model [eqs.1 and 3] can be very well fitted to our experimental data while the unconstrained chromatin model [eqs.1 and 2] does not fit for site separation larger than $35 \mathrm{~kb}$ (Fig. 2a). This confirms that, in both liver cells and $\mathrm{mESC}$, chromatin dynamics in generich TADs undergoes constraints that can be described by polymer models as if, at the supranucleosomal scale, the chromatin was statistically folded into a helix.

However, close examination of best-fit parameters indicates that the statistical helix organization of the chromatin in gene-rich TADs is considerably more relaxed in mESC compared to liver cells (Table 1, compare first and second rows). The mean Pitch $(P)$ of the statistical helix is $201 \pm 13 \mathrm{~nm}$ in $\mathrm{mESC}$ while it is only $160 \pm 9 \mathrm{~nm}$ in liver cells, and the mean diameter $(D)$ is $255 \pm 8 \mathrm{~nm}$ 


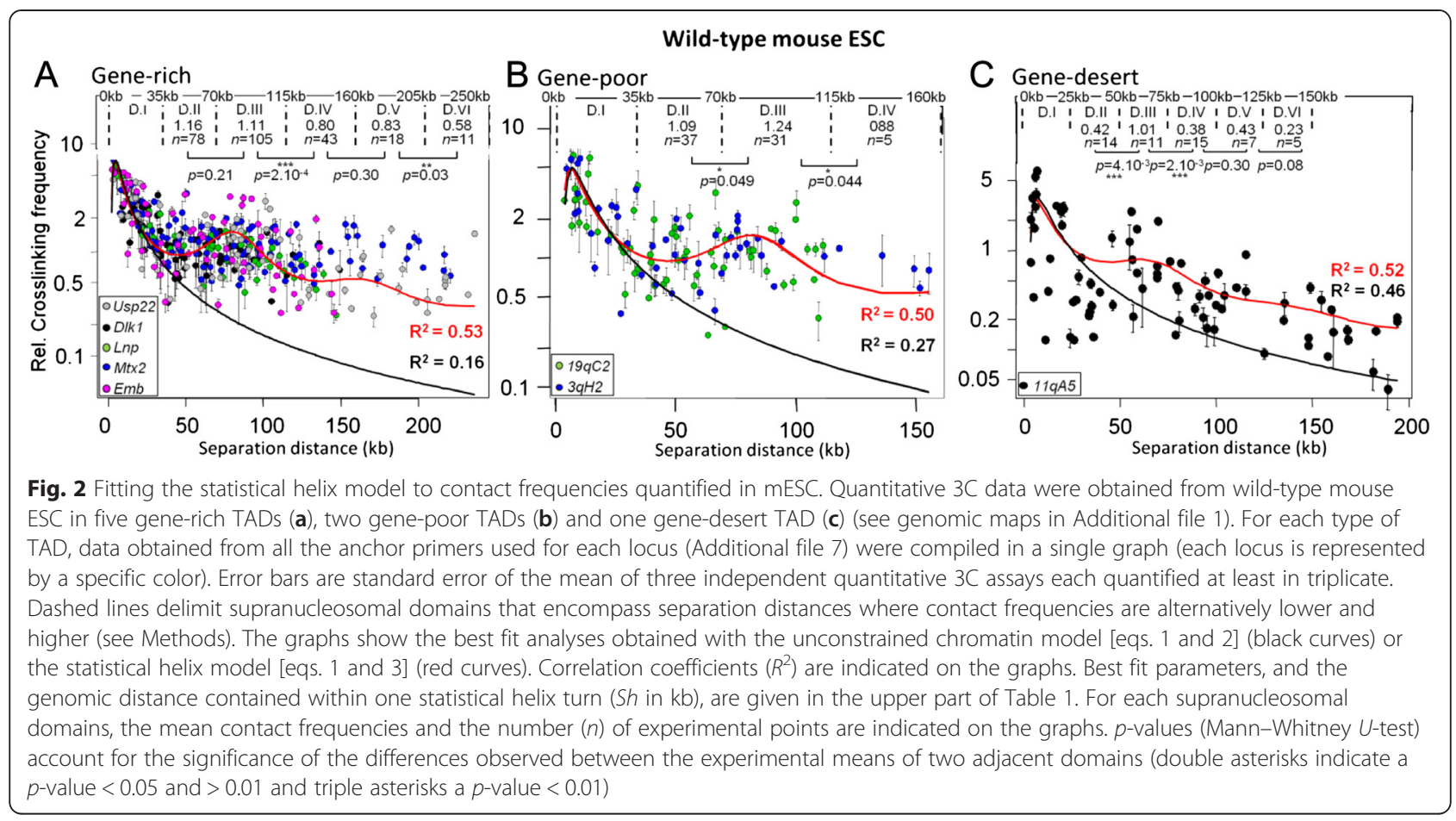

and $287 \pm 5 \mathrm{~nm}$ respectively. Consequently, one turn of the statistical helix $(S h)$ contains $97 \pm 1 \mathrm{~kb}$ of genomic DNA in liver cells while it encompasses only $85 \pm 2 \mathrm{~kb}$ in $\mathrm{mESC}$. Therefore, higher-order chromatin dynamics is less constrained in pluripotent mESC than in liver cells. Remarkably, this clear difference is not linked to local chromatin flexibility since the $S$ parameter is identical $(S=2.7 \pm 0.1 \mathrm{~kb})$ in both cell types (Table 1 , upper part). Finally, the values of the $K$ parameter suggest that cross-linking efficiency is higher in liver cells than in mESC (Table 1, compare first and second rows).

\section{Effects of constraints on chromatin dynamics correlate with gene density in mESC TADs}

Interestingly, inside both gene-poor and gene-desert TADs, chromatin also displayed modulated contact frequencies (see mean contact frequencies in Fig. $2 \mathrm{~b} / \mathrm{c}$ ), indicating that the constraints that impact higher-order chromatin dynamics are present in all genomic contexts investigated. However, while the statistical helix model fits again better than the unconstrained chromatin model to gene-poor TAD data (Fig. 2b), both models could be equally well fitted to the gene-desert data (Fig. 2c), indicating that chromatin dynamics in this latter TAD is not subject to strong constraints. Indeed, the statistical helix is relaxed in gene-desert TADs since one helix turn contains only $72 \mathrm{~kb}$ of genomic DNA while it encompasses more than $85 / 87 \mathrm{~kb}$ in gene-rich or gene-poor TADs (Table 1, compare the fourth row with the second and third rows). Globally, these results indicate that the shape of the statistical helix is progressively more elongated as we go from gene-rich and gene-poor to gene-desert TADs approaching an unconstrained chromatin configuration. Therefore, while the constraints impacting chromatin dynamics can be detected

Table 1 Fitting the statistical helix model to the relative contact frequencies observed in wild-type (upper part, rows 2-4) and triple $\mathrm{KO}$ (lower part, rows 5-7) mouse ES cells (mESCs)

\begin{tabular}{|c|c|c|c|c|c|c|}
\hline & WT vs H1 TKO mESC & $K * 10^{3}$ & $S(\mathrm{~kb})$ & $<D>(\mathrm{nm})$ & $<P>(\mathrm{nm})$ & $S h(k b)$ \\
\hline 1 & mouse liver gene-rich & $890 \pm 70$ & $2.7 \pm 0.1$ & $287 \pm 5$ & $160 \pm 9$ & $97 \pm 1$ \\
\hline 2 & WT mESC gene-rich (Fig. 2a) & $1,070 \pm 80$ & $2.7 \pm 0.1$ & $255 \pm 8$ & $201 \pm 13$ & $85 \pm 2$ \\
\hline 3 & WT mESC gene-poor (Fig. 2b) & $1,880 \pm 360$ & $3.7 \pm 0.3$ & $262 \pm 18$ & $213 \pm 31$ & $87 \pm 5$ \\
\hline 4 & WT mESC gene-desert (Fig. 2c) & $1,380 \pm 370$ & $3.8 \pm 0.3$ & $208 \pm 44$ & $264 \pm 77$ & $72 \pm 13$ \\
\hline 5 & H1TKO gene-rich (Fig. 3a) & $1,810 \pm 140$ & $3.1 \pm 0.1$ & $268 \pm 8$ & $230 \pm 14$ & $83 \pm 2$ \\
\hline 6 & H1TKO gene-poor (Fig. 3b) & $2,270 \pm 430$ & $3.7 \pm 0.3$ & $269 \pm 19$ & $224 \pm 30$ & $83 \pm 5$ \\
\hline 7 & H1TKO gene-desert (Fig. 3c) & $2,620 \pm 840$ & $3.9 \pm 0.4$ & $264 \pm 105$ & $380 \pm 162$ & $86 \pm 28$ \\
\hline
\end{tabular}

Parameters obtained for mouse liver cells [4] are indicated for comparisons (row 1). Remarkable values are indicated in bold (see text) 
in all genomic contexts investigated, their effects are clearly stronger in gene-rich and in gene-poor than in gene-desert TADs.

The models also show that, at the nucleosomal scale, the chromatin is much less flexible in gene-poor $(S=3.7 \pm$ $0.3 \mathrm{~kb})$ and gene-desert $(S=3.8 \pm 0.3 \mathrm{~kb})$ TADs than in gene-rich TADs $(S=2.7 \pm 0.1 \mathrm{~kb})$ (Table 1, compare the third and fourth rows with the second row). However, these changes in chromatin flexibility do not necessarily translate into changes in higher-order chromatin dynamics. Indeed, gene-poor and gene-desert TADs have similar flexibility but different statistical helix organization: one helix turn encompasses $85 / 87 \mathrm{~kb}$ of genomic DNA in gene-poor TADs but only $72 \mathrm{~kb}$ in the gene-desert TAD (Table 1, compare third and fourth rows). Conversely, gene-rich and genepoor TADs have different chromatin flexibility but very similar statistical helix: Pitch $(P)$ is around $200 \mathrm{~nm}$, diameter $(D)$ is about $250 \mathrm{~nm}$ and one helix turn encompasses $85 / 87 \mathrm{~kb}$ of genomic DNA (Table 1, compare second and third rows). Finally, as we noted above, the statistical helix in gene-rich TADs is in a much more open configuration in $\mathrm{mESC}$ than in liver while chromatin flexibility is identical in both cell types (Table 1, compare first and second rows). Therefore, the variations of the higher-order chromatin dynamics observed in vivo in different genomic contexts appear to be largely independent of chromatin flexibility.

\section{Histone $\mathrm{H} 1$ depletion alters chromatin flexibility but not statistical helix organization}

To ascertain that variations of chromatin flexibility do not necessarily impact higher-order chromatin dynamics, we performed quantitative $3 \mathrm{C}$ experiments in mESC that are Triple Knock-Out (H1 TKO) for histone H1 genes H1c, H1d and H1e [22]. Indeed, since it binds between nucleosomes, the linker histone $\mathrm{H} 1$ is thought to be a major factor regulating chromatin compaction and flexibility at the nucleosomal scale [23, 24], but a precise evaluation of its potential role for chromatin dynamics at the supranucleosomal scale is missing. Its depletion should thus allow us to assess whether altering chromatin stiffness will impact higher-order chromatin dynamics. Mice lacking the H1c, H1d and H1e die during embryonic development, but H1 TKO mESC lines can be established, which bear various chromatin structure changes [22]. Identical experiments as described above were thus performed in H1 TKO mESC (Fig. 3) and best-fit parameters of the statistical helix model were obtained for each category of TADs (Table 1, lower part).

In both gene-poor and gene-desert TADs (Fig. 3b and c respectively), where histone $\mathrm{H} 1$ density is very high [25], identical results were obtained in both $\mathrm{H} 1 \mathrm{TKO}$ and wildtype (WT) mESC (Table 1, compare third with sixth rows and fourth with seventh rows respectively). In these TADs, histone $\mathrm{H} 1$ depletion was apparently not sufficient to alter chromatin flexibility. One can note, however, that the values of the $K$ parameter are higher in H1 TKO than in WT mESC (Table 1, compare third with sixth rows and fourth with seventh rows) indicating that cross-linking efficiency is lower upon partial histone H1 depletion.

In gene-rich TADs (Fig. 3a), where histone $\mathrm{H} 1$ density is lower [25] histone $\mathrm{H} 1$ depletion in mouse mESC resulted in a very significant decrease in chromatin flexibility compared to WT mouse $\operatorname{mESC}(S=3.1 \pm 0.1 \mathrm{~kb}$ and $2.7 \pm 0.1 \mathrm{~kb}$ respectively) (Table 1, compare fifth and second rows). This result is in agreement with previous finding indicating that the stiffness of a disordered and poorly condensed chromatin fiber (as in H1 TKO mESC) is large, being directly influenced by the high stiffness of the embedded DNA stretch, while a more organized and condensed fiber (as in WT mESC) is far more flexible [26], provided that nucleosome stacking does not occur (as in gene-deserts where histone $\mathrm{H} 1$ density is very high) [27]. However, despite the significant decrease in chromatin flexibility observed in gene-rich TADs, the parameters of the statistical helix (diameter $D$, pitch $P$, DNA in helix turn $S h$ ) were not significantly altered. The shape of the statistical helix tends to be slightly more elongated in H1 TKO mESC than in WT mESC, but this apparent tendency is not sufficiently strong to be considered as really significant. Therefore, the results presented in Fig. 3a demonstrate that altering chromatin flexibility at the nucleosomal scale in gene-rich TADs, where the statistical helix is prominent, does not necessarily impact significantly the higher-order chromatin organization of these regions.

This indicates that chromatin dynamics at the nucleosomal and supranucleosomal scales are somewhat uncoupled, suggesting that the constraints imposed on higher-order chromatin dynamics within TADs may not necessarily rely on intrinsic local features of the chromatin, like the presence of $\mathrm{H} 1$ linker histone or histone epigenetic modifications, which would affect its nucleosomal organization and oligonucleosome compaction [22]. Therefore, this raises the question of the role of the epigenetic landscapes on chromatin dynamics.

\section{Higher-order chromatin dynamics within Drosophila TADs is unconstrained}

To investigate the influence of the epigenetic contexts on chromatin dynamics, we generated and used $\mathrm{Hi}-\mathrm{C}$ data from the fly Drosophila melanogaster for which epigenetic domains have been extensively described [6]. The Drosophila genome is relatively small in size allowing ultra-high genomic resolution of chromatin contacts. Five billion paired-end $\mathrm{Hi}-\mathrm{C}$ reads were obtained from late Drosophila embryos [28] and normalized Hi-C data were processed in order to produce thousands of "virtual $3 C^{C}$ " profiles 

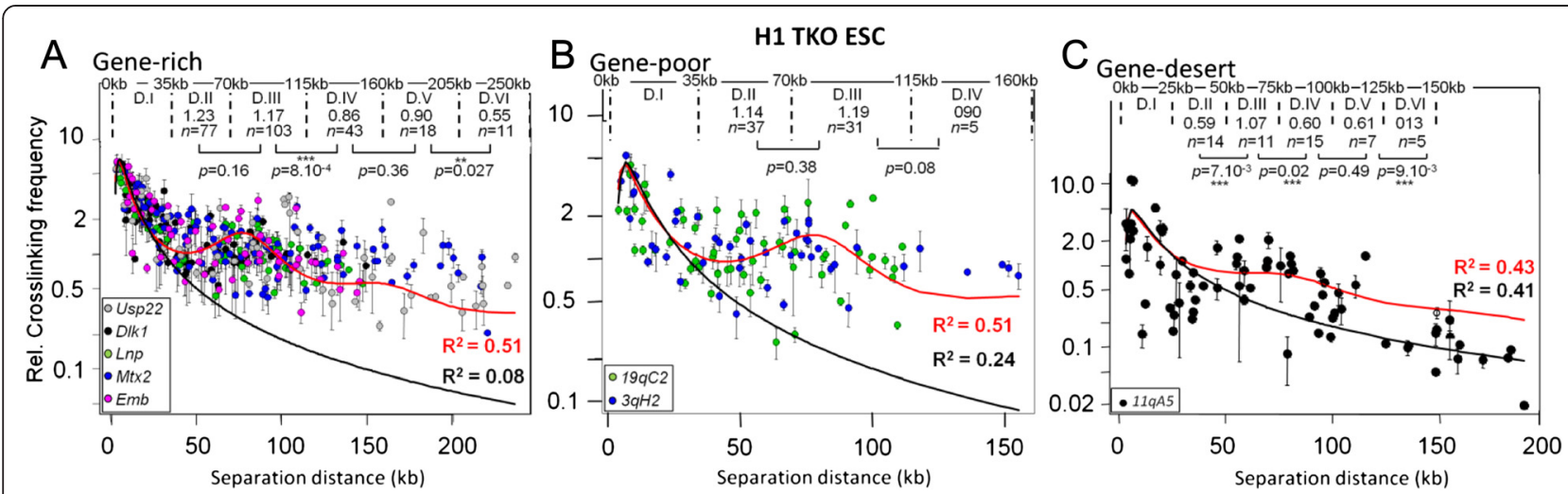

Fig. 3 Fitting the statistical helix model to contact frequencies quantified in mouse H1 TKO ESC. Quantitative $3 C$ data were obtained from mouse ESC that are Triple Knock-Out for Histone $\mathrm{H} 1$ genes (H1 TKO), for five gene-rich TADs (a), two gene-poor TADs (b) and one gene-desert TAD (c). The graphs show the best-fit analyses obtained with the unconstrained chromatin model [eqs. 1 and 2] (black curves) or the statistical helix model [eqs. 1 and 3] (red curves). The data (see Additional file 8) were analyzed and are depicted as described in the legend of Fig. 2. Best-fit parameters, and the genomic distance contained within one statistical helix turn (Sh in kb), are given in the lower part of Table 1

providing relative contact frequencies at $5 \mathrm{~kb}$ resolution throughout the Drosophila genome (see Methods).

To check whether some constraints impact chromatin dynamics in the Drosophila, we first focused our analyses on a subset of "virtual-3C" profiles spanning separation distances of at least $65 \mathrm{~kb}$ without crossing any TAD borders. Among the 2236 "virtual-3C" profiles that could be appropriately fitted to the unconstrained chromatin model [eqs. 1 and 2] $\left(0<\mathrm{R}^{2}<1\right), 66 \%$ had a correlation coefficient $\left(R^{2}\right)$ above 0.5 . This result indicates that the unconstrained chromatin model fits appropriately to most "virtual 3C" profiles and thus, in contrast to previous observation made in mammals [4] (Fig. 2), chromatin dynamics within Drosophila TADs appears as globally unconstrained, and hence non-helical, at the scale of several tens of kilo-bases.

\section{Local properties of Drosophila chromatin are finely tuned according to the epigenetic landscape}

"Virtual 3C" generated were then classified according to chromosomal location and to the previously defined epigenomic domains (D1 to D4) [6]: D1 ("red chromatin") corresponds to domains with "active" epigenetic marks, D2 ("black chromatin") displays no specific epigenetic modifications, D3 ("blue chromatin") is Polycomb (PcG) associated chromatin and D4 ("green chromatin") is HP1/heterochromatin. Finally, for each "virtual 3C", the unconstrained chromatin model was fitted and the three best-fit parameters were extracted (see Additional file 2 for representative examples). For each chromosome, statistical analyses of best-fit parameters were performed separately according to the epigenetic domains.

Box-plots in Fig. 4 show the results of statistical analyses of best-fit parameters obtained for chromosome $2 \mathrm{~L}$. We found that "active" domains (D1, "red chromatin") are less compact (median value of $L$ parameter $=10.81 \mathrm{~nm} /$ $\mathrm{kb})$, more efficiently cross-linked (median value of $K$ parameter $=0.85$ ) and more flexible (median value of $S$ parameter $=4.15 \mathrm{~kb})$ than the other domains $(L=$ $10.56 / 10.66 / 10.32 \mathrm{~nm} / \mathrm{kb}$ for $\mathrm{D} 2 / \mathrm{D} 3 / \mathrm{D} 4$ respectively while $K=1.49 / 1.34 / 2.40$ and $S=4.92 / 4.84 / 5.30 \mathrm{~kb}$ for D2/D3/D4 respectively) (Fig. 4). As expected, we found that HP1/heterochromatin (D4) is much less flexible and more compact than any other type of chromatin. However, "black" (D2) and PcG (D3) chromatins have very similar flexibility and compaction, suggesting that PcG proteins do not significantly impact on local chromatin dynamics (Fig. 4). Identical results were found for all the other Drosophila chromosomes, except for the tiny chromosome 4, which displayed quite flexible and poorly compacted chromatin despite being entirely heterochromatic (Table 2) (full data are in Additional file 3. Additional file 5 gives Wilcox $p$-values of differences observed between the different epigenetic domains for parameters shown in Table 2). This finding is consistent with a recent work demonstrating that chromosome 4 displays distinct epigenetic profiles compared to both pericentric heterochromatin and euchromatic regions and that enrichment of HP1a on chromosome 4 genes creates an alternate chromatin structure which is critical for their regulation [29]. Globally, these experiments confirm that the epigenetic contexts influence significantly the local chromatin dynamics in vivo. However, quantitatively, their effects on chromatin compaction and flexibility appear as being quite limited. Indeed, the largest variations observed (between the "active" and HP1/heterochromatin domains) for chromatin compaction and flexibility are 10.76 to $9.99 \mathrm{~nm} / \mathrm{kb}$, i.e. about $7 \%$, on chromosome $2 \mathrm{R}$, and 4.090 to $5.382 \mathrm{~kb}$, i.e. about $24 \%$, on chromosome $3 \mathrm{~L}$, respectively (Table 2 ). Therefore, the epigenetic 


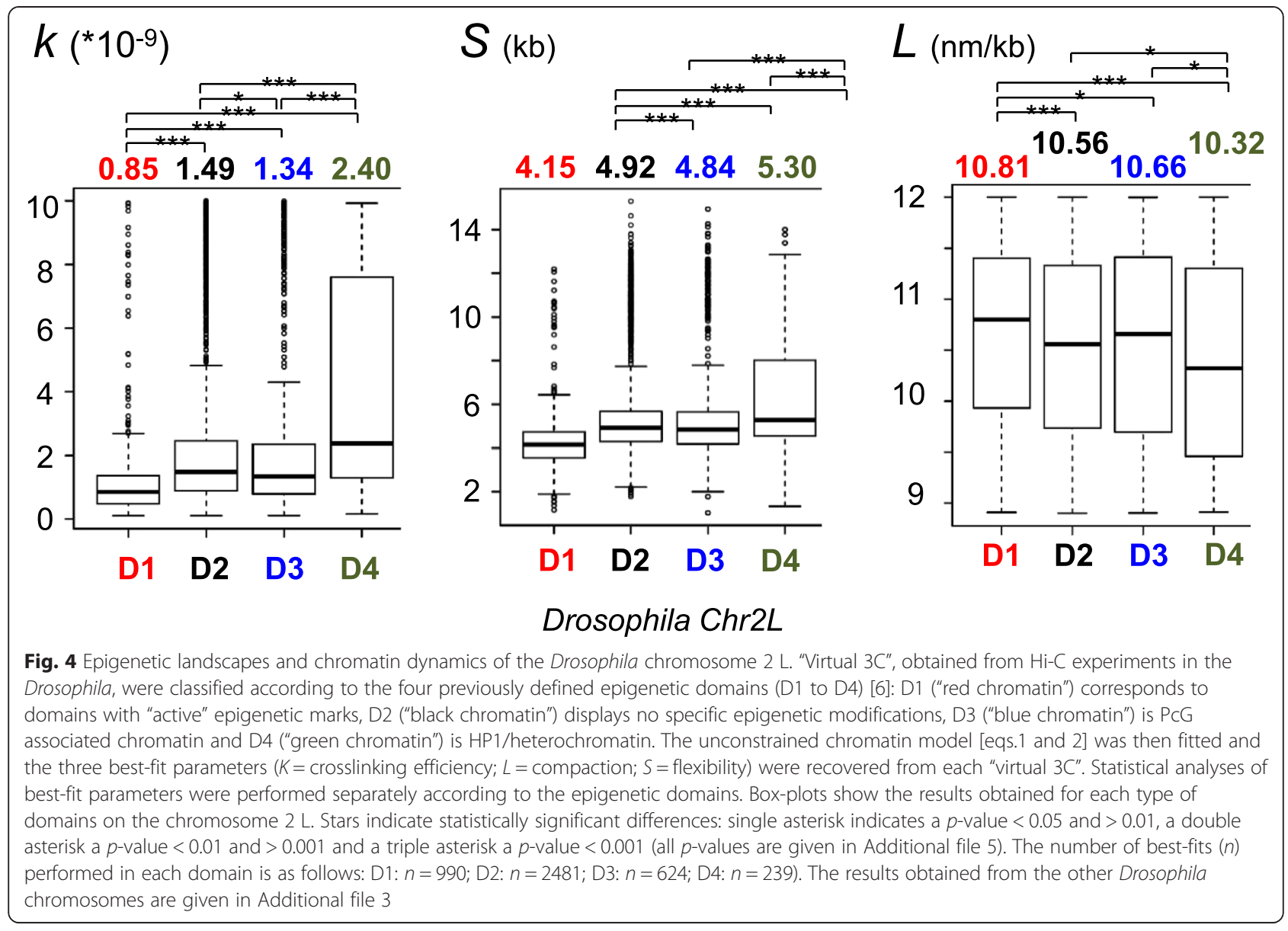

landscape in the fly appears to be involved in finetuning the local chromatin dynamics.

\section{Discussion}

Modulated contact frequencies, the statistical helix and their relevance for genome functions

Our work shows that, in the mouse, a modulation in contact frequency over large genomic distances can be detected in all the three genomic contexts investigated: gene-rich, gene-poor and gene-desert TADs. This demonstrates that the constraints responsible for the emergence of the statistical helix apply widely to the mammalian genome (Fig. 2; Table 1, upper part). However, their effects on higher-order chromatin dynamics are progressively attenuated as we shift from gene-rich and gene-poor to gene-desert TADs where, in this latter case, an unconstrained polymer model can be fitted appropriately to contact frequency data (Fig. 2; Table 1, upper part). This situation is reminiscent to experiments performed in the yeast Saccharomyces cerevisiae [21] where the unconstrained model could be fitted appropriately in AT-rich regions while the statistical helix model provides better fits in GC-rich regions [4].

Furthermore, the statistical helix organization, and its underlying dynamics, seems to be finely tuned according to the cell-type. Indeed, chromatin appears to be less constrained in mESC than in mouse liver cells (the statistical helix is more "elongated" in $\mathrm{mESC}$ ) (Table 1, upper part). This finding is in agreement with several pieces of evidence indicating that, in $\mathrm{mESC}$, chromatin is characterized by an abundance of active chromatin marks [30, 31] and that it displays less compact heterochromatin domains $[30,32,33]$. Therefore, the configuration of the genome makes it more accessible in $\mathrm{mESC}$ than in differentiated cells. It is assumed that this specific chromatin organization is essential to establish pluripotency by maintaining the genome in an open, readily accessible state, allowing for maximum plasticity [16].

"Virtual 3C" profiles reconstructed from $5 \mathrm{C}$ data obtained in $\mathrm{mESC}[10]$ also shows the presence of a very significant modulation in contact frequencies in a $572 \mathrm{~kb}$ gene-poor region displaying no apparent locus-specific interaction (chrX:102,338,477-102,910,171) (Additional file 4). Interestingly, here again, the statistical helix model fits better to these data $\left(R^{2}=0.52\right)$ than the unconstrained chromatin model $\left(R^{2}=0.40\right)$. Therefore, $5 \mathrm{C}$, as well as quantitative $3 \mathrm{C}$ data (Fig. 2), are able to evidence, in $\mathrm{mESC}$, a long-range modulation in contact frequencies which is best described by the statistical helix model. 
Table 2 Fitting the unconstrained model on Drosophila Hi-C data $^{a}$

\begin{tabular}{llllll}
\hline Chromosome & Parameters & Active & Black & PcG & Centromeric \\
& & D1 & D2 & D3 & D4 \\
\hline \multirow{3}{*}{ Chr2L } & $\mathrm{K}^{*} 10^{9}$ & 0.852 & 1.487 & 1.340 & 2.405 \\
& $\mathrm{~S}(\mathrm{~kb})$ & 4.150 & 4.918 & 4.849 & 5.296 \\
& $\mathrm{~L}(\mathrm{~nm} / \mathrm{kb})$ & 10.81 & 10.56 & 10.6 & 10,32 \\
Chr2R & $\mathrm{K}^{*} 10^{9}$ & 0.847 & 1.471 & 1.122 & 2.699 \\
& $\mathrm{~S}(\mathrm{~kb})$ & 4.147 & 4.925 & 4.552 & 5.292 \\
& $\mathrm{~L}(\mathrm{~nm} / \mathrm{kb})$ & 10.76 & 10.57 & 10.71 & 9.99 \\
Chr3L & $\mathrm{K} * 10^{9}$ & 0.808 & 1.383 & 1.232 & 2.684 \\
& $\mathrm{~S}(\mathrm{~kb})$ & 4.090 & 4.881 & 4.873 & 5.382 \\
& $\mathrm{~L}(\mathrm{~nm} / \mathrm{kb})$ & 10.80 & 10.55 & 10.62 & 10.06 \\
Chr3R & $\mathrm{K}{ }^{*} 10^{9}$ & 0.857 & 1.548 & 1.324 & \\
& $\mathrm{~S}(\mathrm{~kb})$ & 4.132 & 4.95 & 4.773 & \\
& $\mathrm{~L}(\mathrm{~nm} / \mathrm{kb})$ & 10.80 & 10.59 & 10.64 & \\
& $\mathrm{~K} * 10^{9}$ & & & & 1.303 \\
& $\mathrm{~S}(\mathrm{~kb})$ & & & & 4.55 \\
& $\mathrm{~L}(\mathrm{~nm} / \mathrm{kb})$ & & & & 10.67 \\
\hline
\end{tabular}

Median values of 3 best-fit parameters obtained on the autosomal chromosomes in each type of TADs

${ }^{\mathrm{a}} \mathrm{Hi}-\mathrm{C}$ samples were prepared from unsexed flies, and therefore the $\mathrm{X}$ chromosome was not analysed since, in males, this chromosome undergoes dosage compensation that largely affects its epigenetic features

As previously indicated [4], the existence of a modulation in contact frequencies has important functional implications, at least in the gene-rich TADs where it is prominent. Indeed, locus-specific functional interactions in these TADs necessarily occur from this underlying dynamics of the chromatin. Therefore, any constraints favouring intrinsically the probability of contact between two genomic regions will also favour the probability of interaction between the regulatory elements that they contain. Long-range interactions should thus tend to occur at preferred relative separation distances where the probability of contact is the highest. We previously showed that, in loci containing co-expressed genes, conserved elements (UCSC database) are overrepresented at a distance of $\sim 100 \mathrm{~kb}$ from the surrounding Transcriptional Start Sites (TSS) [4]. In the same line, ChIP-seq experiments at 885 loci containing genes overexpressed in the mouse forebrain showed that p300 peaks linked to enhancer activities are more significantly enriched for separation distances of about 70 to $80 \mathrm{~kb}$ from the nearest TSS [34]. Finally, extensive 5C experiments focusing on the ENCODE pilot project regions (representing $1 \%$ of the human genome) have recently shown that long-range interactions between TSS and distal elements display a marked asymmetry with a bias for interactions with elements located about $120 \mathrm{~kb}$ upstream of the TSS [35]. Altogether, these observations are in agreement with the existence of a long-range $(\sim 100 \mathrm{~kb})$ modulation of contact frequencies in
gene-rich-TADs, suggesting that the constraints governing statistical helix organization underlie higher-order chromatin dynamics of a very significant part of the genome.

\section{Simple polymer-physics principles govern chromatin dynamics within TADs}

In addition to polymer models as those used in the present work, several other physical models, like the equilibrium and crumpled/fractal globule models, have been developed to describe chromatin dynamics in vivo [20]. Crumpled globule features are characteristic of chromatin dynamics above $1 \mathrm{Mb}$ (chromosome territory/ inter-TADs dynamics) [8]. However, at that scale, simple polymer-physic models, like the "strings and binders switch" (SBS) model [36], can also reproduce crumpled globule conformations, and finally globule features of chromatin organization within TADs remain unexplored. Using quantitative $3 \mathrm{C}$ data (Fig. 2), we showed that, in the absence of any strong locus-specific interaction, contact profiles obtained in gene-rich TADs (Fig. 1) follow a power-law scaling associated to a slope of $-1 / 2$. A similar value has been described for mitotic chromosomes for separation distances encompassing $40 \mathrm{~kb}$ to $10 \mathrm{Mb}$ [12]. However, our samples are devoid of mitotic chromosomes (interphasic nucleus preparations) and therefore, as previously suggested for distances shorter than $100 \mathrm{~kb}$ [8], the contact profiles observed in gene-rich TADs are incompatible with the equilibrium or crumpled globule models. In contrast, they are in good agreement with a more compact conformation as suggested by the SBS model [36]. Therefore, our work reinforce the idea that simple polymerphysics models of chromatin are sufficient to describe chromatin dynamics in vivo $[4,37]$ and it shows that such models and principles also apply within TADs both in mammals and in the fly Drosophila melanogaster. Importantly, neither the equilibrium or crumpled globule models nor the "unconstrained chromatin" model, or so far any other known globule or polymer models, including the SBS model, are able to describe the discrete modulation in contact frequencies that we consistently observed within mammalian TADs in diverse experimental and cellular contexts (3C data in Fig. 2; 5C data in Additional file 4; [4]). Only the statistical helix model is able to account for this feature and it is thus, so far, the simplest model to accurately describe the fundamental chromatin dynamics observed within mammalian TADs. However, this model is clearly not sufficient to describe chromatin dynamics when significant locus-specific interactions take place and, in such conditions, more complex polymer models may indeed be required, taking into account chromatin contacts with nuclear compartments and/or attachment of diffusible factors to binding sites on the chromatin [37]. 
Finally, while the existence of modulated contact frequencies has important implications for chromatin dynamics in a cell population, its interpretation as a helical organization may be far from the reality of an individual conformation at a given time in a single cell. One can note, however, that this model may also be valid to describe chromatin dynamics at the single cell level if the ergodicity of the fluctuations could be verified (i.e. if the average fluctuations observed at a given time in a cell population can recapitulate the average fluctuations over time of an individual conformation).

\section{Conclusion}

Two general types of constraints could contribute to the emergence of the statistical helix organization frequencies within mammalian TADs: "bottom-up" constraints, inherent to some intrinsic constituents of the chromatin, or "top-down" constraints imposed by higher-order superstructures, like chromosome territories and TADs. Despite its remarkable impact on chromatin flexibility in gene-rich TADs, histone $\mathrm{H} 1$ depletion does not significantly affects statistical helix parameters in mESC (Fig. 3; Table 1, lower part). This indicates that chromatin dynamics at the nucleosomal and supranucleosomal scales could be somewhat uncoupled, suggesting that the constraints imposed on higher-order chromatin dynamics during the interphase may not necessarily rely on intrinsic factors of the chromatin that would affect its nucleosomal organization ("bottom-up" constraints).

$\mathrm{Hi}-\mathrm{C}$ data have shown that contact frequencies across TAD borders are extremely low [7]. The statistical helix organization observed in mammals is thus necessarily confined within TADs and cannot extend throughout TAD borders. It is therefore tempting to speculate that, in mammals, TADs borders may represent "top-down" constraints impacting chromatin dynamics at higherorder levels by restricting the space that the chromatin could possibly explore at that scale, thus contributing to the emergence of the statistical helix organization. However, this hypothesis is challenged by the fact that no such constraints are observed in Drosophila TADs.

How to explain such a difference between these two organisms? Rather than speculating that genome organization principles are intrinsically different (which would appear unlikely for two metazoans), it seems more realistic to postulate that the underlying organization principles are similar, but that constraints applied to higher-order chromatin dynamics have different impacts because of distinct critical features of TAD organization in these two organisms. Indeed, Drosophila TADs display a median size of $70 \mathrm{~kb}$ [6] which is considerably smaller than that of mammalian TADs. With a median size of more than $800 \mathrm{~kb}$ [7], mammalian TADs are more prone to constraints that impact chromatin dynamics at higher-order levels i.e. over large genomic distances. Therefore, we propose that, beyond locus-specific interactions, higher-order chromatin dynamics in higher eukaryotes may also rely on "top-down" constraints whose effects are depending on the exact size and organization of the TADs.

\section{Methods \\ Mouse breeding}

All experimental designs and procedures are in agreement with the guidelines of the animal ethics committee of the French "Ministère de l'Agriculture" (European directive 2010/63/EU).

\section{Cell culture}

$\mathrm{mESC}$ were cultured in serum/LIF conditions as previously described [22].

\section{Quantitative 3C / SybGreen assays}

$3 \mathrm{C}$ assays were performed from nucleus preparations as previously described [3, 38, 39]. 3C products were quantified (during the linear amplification phase) on a LighCycler $480 \mathrm{II}$ apparatus (Roche) $\left(10 \mathrm{~min}\right.$. at $95{ }^{\circ} \mathrm{C}$ followed by 45 cycles $10 \mathrm{~s}$. at $95{ }^{\circ} \mathrm{C} / 8$ s. at $69{ }^{\circ} \mathrm{C} / 14 \mathrm{~s}$. at $72{ }^{\circ} \mathrm{C}$ ) using the Hot-Start Platinum ${ }^{\circ}$ Taq DNA Polymerase from Invitrogen (10966-034), the GoTaq ${ }^{\circ}$ Hot-Start Polymerase from Promega (M5005) and a standard 10X qPCR mix [40] where the usual $300 \mu \mathrm{M}$ dNTP have been replaced by $1500 \mu \mathrm{M}$ of CleanAmp dNTP (Tebu-bio $040 \mathrm{~N}-9501-10)$. Standards curves for qPCR have been generated from BACs (RP serie from Invitrogen) as previously described [4]: RP23 $55 \mathrm{I} 2$ for the Usp 22 locus; RP23 117C15 for the Dlk1 locus; RP23 463 J10 and RP23 331E7 for the Lnp locus; RP23 117 N21 for the Mtx2 locus; RP23 $131 \mathrm{E} 7$ for the Emb locus; RP23 30H4 and RP23 247C7 for the $3 \mathrm{qH} 2$ and $19 \mathrm{qC} 2$ gene-poor regions respectively; and a sub-clone derived from RP23 3D5 for the 11qA5 genedesert region (also see Additional file 1a). Quantitative 3C primers sequences are given in Additional file 6 . Data obtained from these experiments are included in Additional file 7 (WT mESC) and Additional file 8 (H1 TKO mESC). The number of sites analysed in each experiment were as follows (Additional file 1b). For WT mESC: Usp22 locus, for anchor sites F1 and F7, 33 and 35 sites were analysed respectively; Dlk1 locus, for anchor sites F3/F5/F14 and F16, 9/16/21 and 26 sites were analysed respectively; Emb locus, for anchor sites R4 and R7, 30 sites were analysed for each anchor; Lnp locus, for anchor site R35, 49 sites were analysed; Mtx2 locus, for anchor sites R2 and R56, 52 and 50 sites were analysed respectively; 3qH2 gene-poor locus, for anchor sites $\mathrm{R} 6$ and R27, 25 sites were analysed for each anchor; $19 q C 2$ gene-poor locus, for anchor sites R41 and R59, 33 sites were analysed for each anchor, and for the 11qA5 genedesert locus, for anchor sites F5/F25/F35 and F48, 21/ 
20/21 and 20 sites were analysed respectively. For H1 TKO mESC: Usp22 locus, for anchor sites F1 and F7, 33 and 34 sites were analysed respectively; $D l k 1$ locus, for anchor sites F3/F5/F14 and F16, 9/16/21 and 24 sites were analysed respectively; $E m b$ locus, for anchor sites R4 and R7, 29 and 30 sites were analysed respectively; Lnp locus, for anchor site R35, 49 sites were analysed; Mtx2 locus, for anchor sites R2 and R56, 52 and 49 sites were analysed respectively; $3 \mathrm{qH} 2$ gene-poor locus, for anchor sites R6 and R27, 25 sites were analysed for each anchor; 19qC2 gene-poor locus, for anchor sites R41 and R59, 33 sites were analysed for each anchor, and for the 11qA5 gene-desert locus, for anchor sites F5/F25/F35 and F48, 18/20/21 and 19 sites were analysed respectively.

\section{Supranucleosomal domains}

The supranucleosomal domains (D.I to D.VI) encompass separation distances where random collision frequencies are alternatively lower and higher; They were assessed by statistical analyses (Mann-Whitney U tests) performed on data shown in Figs. 2 and 3. For gene-rich and genepoor loci : 0 to $35 \mathrm{~kb}$ (domain I), 35-70 kb (domain II), 70-115 kb (domain III), 115-160 kb (domain IV), 160$205 \mathrm{~kb}$ (domain V) and 205-250 kb (domain VI). For the gene-desert region : 0 to $25 \mathrm{~kb}$ (domain I), 25-50 kb (domain II), 50-75 kb (domain III), 75-100 kb (domain IV), 100-125 kb (domain V) and 125-150 kb (domain VI).

\section{Mathematical methods}

We used a model that combines the Freely Jointed Chain/Kratky-Porod worm-like chain models as described in reference [41]. This combined model (equation 3 of reference [21]), which expresses the relation between the cross-linking frequency $X(s)$ (in mol $\mathrm{x} \mathrm{liter}^{-1} \mathrm{x} \mathrm{nm}^{3}$ ) and the site separation $s$ (in $\mathrm{kb}$ ) along the genome, is as follows:

$$
X(s)=\left[K \times 0.53 \times \beta^{-3 / 2} \times \exp \left(-{ }^{2} / \beta^{2}\right) \times(L \times S)^{-3}\right]
$$

with, for an unconstrained polymer:

$$
\left.\beta={ }^{s} / s \text { (unconstrained chromatin model }\right)
$$

In equation [1], the linear mass density $L$ is the length of the chromatin in $\mathrm{nm}$ that contains $1 \mathrm{~kb}$ of genomic DNA. We used different $L$ values estimated from a packing ratio of 6 nucleosomes per $11 \mathrm{~nm}$ of chromatin in solution at physiological salt concentrations $[42,43]$ and a nucleosome repeat length (NRL) of 194 base pairs as found in mouse liver [44] or NRL = 189 and 174 nt for wild-type and TKO mESC respectively [22]. This led to values of $L=9.45 \mathrm{~nm} /$ $\mathrm{kb}$ for mouse liver cells, $L=9.70 \mathrm{~nm} / \mathrm{kb}$ for $\mathrm{mESC}$ and
$L=10.53 \mathrm{~nm} / \mathrm{kb}$ for TKO mESC. $S$ is the length of the Kuhn's statistical segment in kb, which is a measure for the flexibility of the chromatin. The parameter $K$ represents the efficiency of cross-linking which reflects experimental variations [1].

We previously showed that mammalian chromatin undergoes constraints that results in a modulation of contact frequencies along some regions of the chromatin [4]. This modulation can be described by a specific polymer model, called the statistical helix model, where the following $\beta$ term is used in equation [1] (see ref. [4]):

$$
\beta=\frac{\sqrt{D^{2} \times \sin ^{2}\left[\frac{\pi \times L \times s}{\sqrt{\pi^{2} \times D^{2}+P^{2}}}\right]+\left[\frac{P^{2} \times L^{2} \times s^{2}}{\pi^{2} \times D^{2}+P^{2}}\right]}}{L \times S}(\text { statistical helix model })
$$

where $P$ is the mean Pitch and $D$ the mean diameter in $\mathrm{nm}$ of the statistical helix. The length of one turn on the statistical helix $S h$ in kb (Table 1) was calculated using best-fit parameters and equation [4]:

$$
\mathrm{Sh}=\frac{\sqrt{(\pi \times D) 2+\left(P^{2}\right)}}{\mathrm{L}}(\mathrm{kb})
$$

\section{Best-fit analyses of quantitative $3 C$ data from mouse ECS}

Best-fit analyses were implemented under the $\mathrm{R}$ software ( $\mathrm{R}$ Development Core Team 2008, http://www.R-project.org), as previously described [4]. We used the "nls object" (package stats version 2.8.1) which determines the nonlinear (weighted) least-squares estimates of the parameters of nonlinear models.

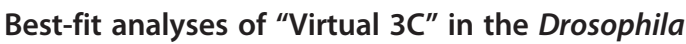 melanogaster}

$\mathrm{Hi}-\mathrm{C}$ data were obtained from total Drosophila embryos and normalized tag numbers were assembled into $5 \mathrm{~kb}$ bins as previously described [6, 28]. Datasets have been submitted to Gene Expression Omnibus (GEO) under accession no [GSE61471] (http://www.ncbi.nlm.nih.gov/ geo/query/acc.cgi?acc=GSE61471). The relative contact frequencies used to construct the "virtual 3C" profiles were obtained by assembling these $5 \mathrm{~kb}$ bins into larger $25 \mathrm{~kb}(5 * 5 \mathrm{~kb})$ bins that were analyzed with a step of $5 \mathrm{~kb}$ along the chromosomes. For each $25 \mathrm{~kb}$ bins, the relative contact frequencies were calculated each $5 \mathrm{~kb}$ within a region surrounding $400 \mathrm{~kb}(80 * 5 \mathrm{~kb}$ bins) from the start of the $25 \mathrm{~kb}$ bin. For each "virtual 3C", the unconstrained chromatin model [eqs. 1 and 2] was fitted to the first $70 \mathrm{~kb}(14 * 5 \mathrm{~kb}$ bins) using the " $n l s 2$ object" under the R software (R Development Core Team 2008, http://www.R-project.org), and the best-fit parameters were extracted. Statistical analyses of these parameters were performed separately on each chromosome and 
according to the type of epigenetic domain (Fig. 2 and Additional file 2). Wilcox $p$-values were calculated to assess the significance of differences observed between the values obtained in each case (Additional files 1 and 9).

\section{Availability of supporting data}

The data set supporting the results of this article is available in the Gene Expression Omnibus repository, [GSE61471, http://www.ncbi.nlm.nih.gov/geo/query/acc.cgi? acc=GSE61471].

\section{Additional files}

Additional file 1: Genomic maps of the TADs investigated in the present study. (a) Map of the TADs containing the loci analyzed in mESC. The Hi-C data (http://yuelab.org/hi-c/database.php) ([1]) are displayed on the top of each map. Gene locations are presented as visualized in the UCSC browser. The black squares in the Hi-C data and the location of the BACs (black bars below the genes) help to demarcate the regions analyzed in our quantitative $3 C$ experiments. Red and green rectangles indicate a negative or a positive directionality index respectively, as defined in ref. [1]. Blue rectangles are located at the borders of each TAD. (b) Detailed map of the loci investigated by quantitative 3 C. Genes are indicated by full boxes and promoters by thick black arrows above these boxes. The scale-bar indicates the size of $10 \mathrm{~kb}$ of sequence. The names of the loci and chromosomal location are indicated above each map. The HindllI (Usp22, Emb, Lnp, Mtx2, 19qC2, $3 \mathrm{qH} 2$ and 11qA5 loci) or EcoRI (DIk1 locus) sites investigated are indicated on the maps. Arrows labeled with a "F" (forward) or a "R" (reverse) indicate the positions of the primers used as anchors in quantitative 3 Cexperiments. The location ( $\mathrm{mm} 9$ ), size (in Mb) and gene density (TSS/Mb) of each TAD investigated are indicated on the right. Note that the very low contact frequencies observed for regions investigated on chromosomes 3 and 11qA5 impair the accurate location of TAD borders. TAD sizes provided here are those determined from data published in ref. [1] (Additional file 9). (PDF $300 \mathrm{~kb}$ )

Additional file 2: Nine examples of fits of the unconstrained chromatin model [eqs. 1 and 2] on "Virtual 3 C » data obtained on Drosophila melanogaster chromosome $\mathbf{2} \mathbf{L}$. Because of the small size of TADs in the Drosophila, the unconstrained chromatin model was fitted to the first $70 \mathrm{~kb}$ of the data. Note that the fit is generally in very good agreement with the unconstrained chromatin model even for larger separation distances. (PDF $250 \mathrm{~kb}$ )

Additional file 3: Epigenetic landscapes and chromatin dynamics of the Drosophila chromosomes. "Virtual 3C" were obtained and analysed from each chromosome as described in Fig. 4. Statistical analyses of best-fit parameters were performed separately according to the epigenetic domains (Note that chromosome 4 is exclusively composed of D4 Domains, while chromosome 3 is devoid of such domains). Box-plots show the results obtained for each type of domains on each chromosome. Chromosome $X$ was excluded from the analyses because dosage compensation affects epigenetic landscapes of this chromosome in males and the embryos used in the Hi-C experiments were not sexed. The number of best-fits ( $n$ ) performed in each domain is as follows: for chromosome 2R, D1: $n=1391 ; \mathrm{D} 2: n=1943 ; \mathrm{D} 3: n=350$; D4: $n=290$, for chromosome $3 \mathrm{~L}, \mathrm{D} 1: n=1096 ; \mathrm{D} 2: n=2650 ; \mathrm{D} 3: n=609$; D4: $n=272$, for chromosome 3R, D1: $n=1525 ; \mathrm{D} 2: n=3197 ; \mathrm{D} 3: n=755$, for chromosome 4, D4: $n=225$. (PDF $260 \mathrm{~kb}$ )

Additional file 4: fitting the statistical helix model to contact frequencies quantified by $5 \mathrm{C}$ experiments in $\mathrm{mECS}$ : (a) $5 \mathrm{C}$ Matrix from data obtained in mESC [2] indicating the $572 \mathrm{~kb}$ gene-poor region (region 1) with no apparent locus-specific interaction (chrX:102,338,477-102,910,171) that we used to fit polymer models. (b) "Virtual 3C" profiles were reconstructed from region 1 and data were compiled in a single graph. Error bars are standard error of the mean of two 5C experiments. Dashed lines delimit supranucleosomal domains that encompass separation distances where contact frequencies are alternatively lower and higher (see Methods). The graph shows the best fit analyses obtained with the unconstrained chromatin model [eqs. 1 and 2] (black curve) or the statistical helix model [eqs. 1 and 3] (red curve). Correlation coefficients (R2) are indicated on the graph. For each supranucleosomal domains, the mean contact frequencies and the number $(n)$ of experimental points are indicated on the graph. $p$-values (Mann-Whitney U-test) account for the significance of the differences observed between the experimental means of two adjacent domains. (PDF $200 \mathrm{~kb}$ )

Additional file 5: Statistical tests. This table gives, for each chromosome and each domain, the Wilcox $p$-values for the differences observed between the median values of the three parameters presented in Table 2 ( $k=$ crosslinking efficiency; $L=$ compaction; $S=$ flexibility). (PDF $72 \mathrm{~kb}$ )

Additional file 6: Quantitative 3C primer sequences. (XLS $56 \mathrm{~kb}$ ) Additional file 7: Quantitative $3 C$ dataset for WT mouse embryonic stem cells. (XLS $89 \mathrm{~kb}$ )

Additional file 8: Quantitative $3 \mathrm{C}$ dataset for $\mathrm{H} 1$ TKO mouse embryonic stem cells. (XLS $86 \mathrm{~kb}$ )

Additional file 9: Additional references. (PDF 108 kb)

\section{Abbreviations}

TAD: Topologically Associating Domains; 3C: Chromosome Conformation Capture; mESC: mouse Embryonic Stem Cells; H1 TKO: Triple Knock-Out of Histone $\mathrm{H} 1$ genes; qPCR: Real-time quantitative polymerase chain reaction.

\section{Competing interests}

The authors declare that they have no competing interests.

\section{Authors' contributions}

VE participated in the design of the study, performed 3C-qPCR experiments and best-fit analyses. TS participated in the design of the study and performed $\mathrm{Hi}-\mathrm{C}$ experiments. TG performed best-fit and statistical analyses of Hi-C data. LH performed 3C-qPCR experiments. MOB contributed analytic tools used for 3C-qPCR primer design. YZ carried out H1 TKO experiments. SB performed 5C data analyses. MNLT and GCa participated in the design of the study and performed 3C-qPCR experiments. AL and JMV participated in the design of the study and contributed to the development of physical models. YF and GC participated in the design of the study and edited the manuscript. TF conceived and designed the study, contributed to the development of physical models, performed best-fit analyses and wrote the manuscript. All authors read and approved the final manuscript.

\section{Acknowledgements}

We thank Hidemasa Kato for stimulating discussions, Françoise Carbonell, Cosette Rebouissou and the staff from the IGMM animal unit for technical assistance. This work was supported by grants from the Institut National du Cancer [PLBIO 2012-129, INCa_5960 to T.F.], the Association pour la Recherche contre le Cancer [SFI20101201555 to T.F.], the Ligue contre le cancer (comité Hérault) and the Centre National de la Recherche Scientifique. Y.F. \& Y.Z. are supported in part by a Georgia Research Alliance Distinguished Cancer Scholar Award (to Y.F.) and by a US National Institutes of Health grant GM085261 (to Y.F.).

\section{Author details}

'Institut de Génétique Moléculaire de Montpellier, UMR5535, CNRS, Université de Montpellier, 1919 Route de Mende, 34293 Montpellier, Cedex 5, France. ${ }^{2}$ Institut de Génétique Humaine, UPR 1142, CNRS, Montpellier, France. ${ }^{3}$ School of Biology and the Petit Institute for Bioengineering and Bioscience, Georgia Institute of Technology, Atlanta, Georgia, USA. ${ }^{4}$ CNRS GDR 3536 UPMC, Sorbonne universités, Paris, France. ${ }^{5}$ Laboratoire de Physique de la Matière Condensée, CNRS UMR 7600, UPMC, Sorbonne universités, Paris, France. 


\section{References}

1. Dekker J, Rippe K, Dekker M, Kleckner N. Capturing chromosome conformation. Science. 2002;295:1306-11.

2. de Wit $E$, de Laat W. A decade of $3 C$ technologies: insights into nuclear organization. Genes Dev. 2012;26:11-24.

3. Hagège $H$, Klous $P$, Braem C, Splinter E, Dekker J, Cathala G, et al. Quantitative analysis of chromosome conformation capture assays (3C-qPCR). Nat Protoc. 2007;2:1722-33.

4. Court F, Miro J, Braem C, Lelay-Taha M-N, Brisebarre A, Atger F, et al. Modulated contact frequencies at gene-rich loci support a statistical helix model for mammalian chromatin organization. Genome Biol. 2011;12:R42.

5. Duan Z, Andronescu M, Schutz K, Mcllwain S, Kim YJ, Lee C, et al. A three-dimensional model of the yeast genome. Nature. 2010;465:363-7.

6. Sexton T, Yaffe E, Kenigsberg E, Bantignies F, Leblanc B, Hoichman M, et al. Three-dimensional folding and functional organization principles of the Drosophila genome. Cell. 2012;148:458-72.

7. Dixon JR, Selvaraj S, Yue F, Kim A, Li Y, Shen Y, et al. Topological domains in mammalian genomes identified by analysis of chromatin interactions. Nature. 2012;485:376-80.

8. Lieberman-Aiden E, van Berkum NL, Williams L, Imakaev M, Ragoczy T, Telling $A$, et al. Comprehensive mapping of long-range interactions reveals folding principles of the human genome. Science. 2009:326:289-93.

9. Rao SS, Huntley MH, Durand NC, Stamenova EK, Bochkov ID, Robinson JT, et al. A 3D Map of the human genome at kilobase resolution reveals principles of chromatin looping. Cell. 2014;159:1665-80.

10. Nora EP, Lajoie BR, Schulz EG, Giorgetti L, Okamoto I, Servant N, et al. Spatial partitioning of the regulatory landscape of the X-inactivation centre. Nature. 2012;485(7398):381-5.

11. Giorgetti L, Servant N, Heard E. Changes in the organization of the genome during the mammalian cell cycle. Genome Biol. 2013;14:142

12. Naumova N, Imakaev M, Fudenberg G, Zhan Y, Lajoie BR, Mirny LA, et al. Organization of the mitotic chromosome. Science. 2013;342:948-53.

13. Van Bortle K, Nichols MH, Li L, Ong CT, Takenaka N, Qin ZS, et al. Insulator function and topological domain border strength scale with architectural protein occupancy. Genome Biol. 2014;15:R82.

14. Zuin J, Dixon JR, van der Reijden MI, Ye Z, Kolovos P, Brouwer RW, et al. Cohesin and CTCF differentially affect chromatin architecture and gene expression in human cells. Proc Natl Acad Sci U S A. 2014;111:996-1001.

15. Andrey G, Montavon T, Mascrez B, Gonzalez F, Noordermeer D, Leleu M, et al. A switch between topological domains underlies HoxD genes collinearity in mouse limbs. Science. 2013;340:1234167.

16. Cavalli G, Misteli T. Functional implications of genome topology. Nat Struct Mol Biol. 2013:20:290-9.

17. Gibcus JH, Dekker J. The hierarchy of the 3D genome. Mol Cell. 2013;49:773-82.

18. Giorgetti L, Galupa R, Nora EP, Piolot T, Lam F, Dekker J, et al. Predictive polymer modeling reveals coupled fluctuations in chromosome conformation and transcription. Cell. 2014;157:950-63.

19. Nobrega MA, Zhu Y, Plajzer-Frick I, Afzal V, Rubin EM. Megabase deletions of gene deserts result in viable mice. Nature. 2004;431:988-93.

20. Mirny LA. The fractal globule as a model of chromatin architecture in the cell. Chromosome Res. 2011;19:37-51.

21. Dekker J. Mapping in vivo chromatin interactions in yeast suggests an extended chromatin fiber with regional variation in compaction. J Biol Chem. 2008;283:34532-40.

22. Fan Y, Nikitina T, Zhao J, Fleury TJ, Bhattacharyya R, Bouhassira EE, et al. Histone $\mathrm{H} 1$ depletion in mammals alters global chromatin structure but causes specific changes in gene regulation. Cell. 2005;123:1199-212.

23. Happel N, Doenecke D. Histone $\mathrm{H} 1$ and its isoforms: contribution to chromatin structure and function. Gene. 2009;431:1-12.

24. Recouvreux P, Lavelle C, Barbi M, Conde E, Silva N, Le Cam E, et al. Linker histones incorporation maintains chromatin fiber plasticity. Biophys J. 2011;100:2726-35.

25. Cao K, Lailler N, Zhang Y, Kumar A, Uppal K, Liu Z, et al. High-resolution mapping of h1 linker histone variants in embryonic stem cells. PLoS Genet. 2013;9:e1003417.

26. Ben-Haim E, Lesne A, Victor JM. Chromatin: a tunable spring at work inside chromosomes. Phys Rev E Stat Nonlin Soft Matter Phys. 2001;64:051921.

27. Wedemann G, Langowski J. Computer simulation of the 30-nanometer chromatin fiber. Biophys J. 2002;82:2847-59.
28. Schuettengruber B, Oded Elkayam N, Sexton T, Entrevan M, Stern S, Thomas A, et al. Cooperativity, specificity, and evolutionary stability of polycomb targeting in Drosophila. Cell Rep. 2014;9:219-33.

29. Riddle NC, Jung YL, Gu T, Alekseyenko AA, Asker D, Gui H, et al. Enrichment of HP1a on Drosophila chromosome 4 genes creates an alternate chromatin structure critical for regulation in this heterochromatic domain. PLOS Genet. 2012;8:e1002954.

30. Meshorer E, Yellajoshula D, George E, Scambler PJ, Brown DT, Misteli T. Hyperdynamic plasticity of chromatin proteins in pluripotent embryonic stem cells. Dev Cell. 2006;10:105-16.

31. Mikkelsen TS, Ku M, Jaffe DB, Issac B, Lieberman E, Giannoukos G, et al. Genome-wide maps of chromatin state in pluripotent and lineagecommitted cells. Nature. 2007:448:553-60.

32. Efroni S, Duttagupta R, Cheng J, Dehghani H, Hoeppner DJ, Dash C, et al. Global transcription in pluripotent embryonic stem cells. Cell Stem Cell. 2008;2:437-47.

33. Fussner E, Djuric U, Strauss M, Hotta A, Perez-Iratxeta C, Lanner F, et al. Constitutive heterochromatin reorganization during somatic cell reprogramming. EMBO J. 2011;30:1778-89.

34. Visel A, Blow MJ, Li Z, Zhang T, Akiyama JA, Holt A, et al. ChIP-seq accurately predicts tissue-specific activity of enhancers. Nature. 2009;457:854-8.

35. Sanyal A, Lajoie BR, Jain G, Dekker J. The long-range interaction landscape of gene promoters. Nature. 2012;489:109-13.

36. Barbieri M, Chotalia M, Fraser J, Lavitas LM, Dostie J, Pombo A, et al. Complexity of chromatin folding is captured by the strings and binders switch model. Proc Natl Acad Sci U S A. 2012;109:16173-8.

37. Barbieri M, Fraser J, Lavitas LM, Chotalia M, Dostie J, Pombo A, et al. A polymer model explains the complexity of large-scale chromatin folding. Nucleus. 2013;4:267-73.

38. Braem C, Recolin B, Rancourt RC, Angiolini C, Barthes P, Branchu P, et al. Genomic matrix attachment region and chromosome conformation capture quantitative real time PCR assays identify novel putative regulatory elements at the imprinted DIk1/Gt/2 locus. J Biol Chem. 2008;283:18612-20.

39. Court F, Baniol M, Hagège $H$, Petit JS, Lelay-Taha M-N, Carbonell F, et al. Long-range chromatin interactions at the mouse lgf2/H19 locus reveal a novel paternally expressed long non-coding RNA. Nucleic Acids Res. 2011;39:5893-906.

40. Lutfalla G, Uzé G. Performing quantitative reverse-transcribed polymerase chain reaction experiments. Methods Enzymol. 2006;410:386-400

41. Rippe K. Making contacts on a nucleic acid polymer. Trends Biochem Sci. 2001;26:733-40.

42. Gerchman SE, Ramakrishnan V. Chromatin higher-order structure studied by neutron scattering and scanning transmission electron microscopy. Proc Natl Acad Sci U S A. 1987;84:7802-6.

43. Ghirlando R, Felsenfeld G. Hydrodynamic studies on defined heterochromatin fragments support a 30-nm fiber having six nucleosomes per turn. J Mol Biol. 2008;376(5):1417-25.

44. Dalal Y, Fleury TJ, Cioffi A, Stein A. Long-range oscillation in a periodic DNA sequence motif may influence nucleosome array formation. Nucleic Acids Res. 2005;33(3):934-45

\section{Submit your next manuscript to BioMed Central and take full advantage of:}

- Convenient online submission

- Thorough peer review

- No space constraints or color figure charges

- Immediate publication on acceptance

- Inclusion in PubMed, CAS, Scopus and Google Scholar

- Research which is freely available for redistribution 